\title{
An effective test case prioritization by combination of strategies
}

\author{
Ehsan Ganjkhani ${ }^{1}$ D $\cdot$ Mohsen Afsharchi ${ }^{1}$
}

(c) Springer Nature Switzerland AG 2019

\begin{abstract}
One of the most important and costly tests in software engineering is regression testing, which is performed after any change made to the software. One way to reduce the cost of this test is using test case prioritization, in which the two strategies of Total and Additional have been of most interest. Each of these two strategies have their own limitations, which causes them not to have stable behavior in different cases. In this paper, by combining these two strategies with the similarity-based approach, one new approach is proposed for prioritizing the test cases. In an empirical study, we have evaluated our method compared to eight other methods on seven $C$ programs. The results demonstrate that the proposed method has the best performance on average and is more stable than other techniques.
\end{abstract}

Keywords Software testing $\cdot$ Regression testing $\cdot$ Test case prioritization

\section{Introduction}

Regression test is performed alongside software development, to make sure that these changes do not infect existing functionalities. Considering the size of the test suite and its constant runs for each change, this type of testing has usually high costs. Therefore, different techniques for optimizing this type of testing has been proposed and test case prioritization (TCP) is one of them [1].

Of all types of strategies that have been proposed for TCP so far, two generic strategies of Total and Additional are two of the main ones $[2,3]$. Beside their simplicity and performance, each of these strategy have their own limitations. For example, in Total approach it is possible that while some parts are covered repeatedly, some other parts are remained not covered and their faults are found at later times. On the other hand, the Additional approach may cover some statements and be unable to detect fault in those statements, and in this way the fault in that part is later discovered. These limitations make each of these strategy unstable regarding their rate of fault detection. These two strategies complement each other [3].
Another strategy among TCP techniques is similaritybased strategy, in which considering various criteria, test cases are decided upon based on their degree of similarity $[4,5]$.

In this paper, combining three strategies of Total, Additional and similarity-based, we proposed a new technique for TCP and conducted an empirical study and compared our proposed method with eight other techniques, three of which are based on Total strategy which are total-statement (TS), total-branch (TB) and total-method (TM) and three are based on Additional strategy which are additional-statement (AS), additional-branch (AB) and additional-method (AM). Also we used two other techniques of Unordered and Optimal [2] and by comparing our method to these two we can evaluate how close we are to the Optimal technique and how far from the Unordered one. To measure how fast a test suite is to detect faults, we used average percentage of faults detected (APFD) [6]. The results demonstrate that our technique has been able to dominate most of the other techniques regarding the fault detection rate. Also among all these eight techniques, our proposed method has had the most stable performance.

Ehsan Ganjkhani, ganjkhaniehsan@gmail.com | 'Department of Computer Engineering, University of Zanjan, Zanjan 45371-38791, Iran.

SN Applied Sciences (2019) 1:1049 | https://doi.org/10.1007/s42452-019-1076-1 


\section{Proposed method}

In our method, we only use the statement and branch coverage alongside a concept called spanning-branches, which is only a subset and a particular type of branches, and is not a new coverage criterion [7]. Our proposed approach is also somewhat like similarity-based techniques. The chosen feature for the similarity between the two test cases that we used in this study is the statement coverage of them.

Apart from the chosen feature for similarity, there are different criteria to measure the similarity, which are called the metric functions of distance [8]. In the proposed method, we use Jaccard distance as a distance function [9].

Algorithm 1 illustrates the algorithm of the proposed method, the input of this algorithm is the initial unordered list (UL) of all test cases. The output of this algorithm will also be a priority list (PL) of the same test cases.

At first, the distance between all test cases in the UL is calculated two by two based on their statement coverage (similarity-based strategy).This distance is measured with Jaccard Distance in the algorithm. If this distance equals zero, that is, the two tests are exactly the same in terms of coverage, one of them remains in the working set (WS) and the other will move to reserved set (RS).

In the next step, each time, among the test cases in the WS, one item is selected based on the maximum coverage of spanning-branches not covered so far, after which the test is added to the PL and removed from the WS (Additional strategy). After selecting and adding each test case to the $\mathrm{PL}$, it is examined if we have reached full coverage on the spanning-branches. Now, if this condition is satisfied, then all the tests remaining in the WS will be sorted in descending order based on their branch coverage, and the algorithm will exit the while loop (Total strategy). Then all the test cases sorted in the WS are added to the PL. By reaching this stage, it's time to append all the test cases that were reserved at the beginning of the algorithm in the RS to the end of PL.

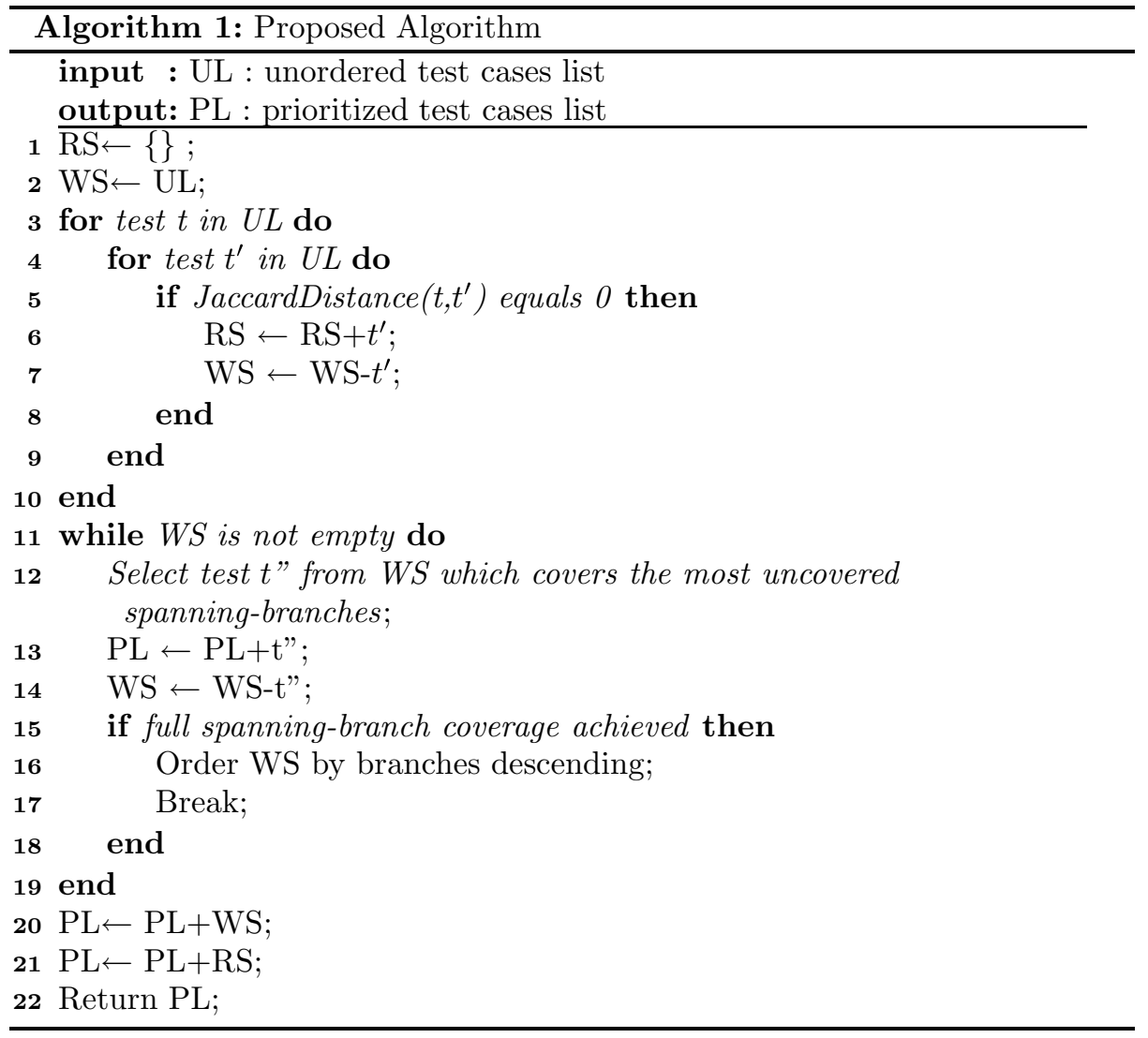




\section{Empirical study}

\subsection{Experiment setup}

We conducted an empirical study to answer the following research questions:

RQ1: Can the proposed method work better than the Total and Additional strategies in most cases regarding fault detection rate?

RQ2: Can the proposed method work better than two strategies of Total and Additional in terms of stability?

We used seven $C$ programs, known as Siemens benchmarks [10, 11], which have been used frequently in empirical studies on regression testing [5, 12-14]. The summary of information about these programs can also be found in Table 1. Then ran our proposed method as well as other eight techniques on these programs then recorded the output results of them as APFD. All experiments in this paper were carried out on a system with Ubuntu 16.04 OS, 6 GB RAM and $2 \mathrm{GHz}$ Intel Celeron processor, and the techniques were also implemented in Java.

\subsection{Results and analysis}

To answer RQ1, we must compare the fault detection rate of each method on each program. This information can be found in Tables 2 and 3. To simplify the comparison and

Table 1 Subject programs

\begin{tabular}{lccc}
\hline Program & \#LoC & \#Faulty versions & \#Test cases \\
\hline Printtokens1 & 726 & 7 & 4130 \\
Printtokens2 & 570 & 9 & 4115 \\
Replace & 564 & 31 & 5542 \\
Schedule1 & 412 & 9 & 2650 \\
Schedule2 & 374 & 8 & 2710 \\
Tcas & 173 & 39 & 1608 \\
Totinfo & 565 & 22 & 1052 \\
\hline
\end{tabular}

viewing of the results, the methods (except for the Optimal), according to their performance in fault detection, are sorted from best to worst in Table 4 .

Obviously, the Optimal method by default has the best performance, so this technique has been ignored in this ranking. As we can see, our method has only been unable to have the best performance in the two programs printtokens1 and printtokens2, but it has the best performance in other programs. Therefore, our proposed method is better than the Total and Additional strategies in most cases, in terms of fault detection rate.

None of the two strategies of Total and Additional have been able to show stable performances. On the other hand, according to Table 4, our proposed method, in the worst case, has not been able to perform best only in two cases including printtokens 1 and printtokens 2 .

It should be noted that according to Tables 2 and 3 , the difference in the result of the proposed method with the best method (TS) regarding to these two programs is close to 0.0001 , which is really insignificant (but considering the number of test cases in these two programs this value cannot be ignored). This fact suggests that the proposed method has a good stability and does not have much fluctuation in its behavior in the various programs, and if it is not the best, at least it could be ranked second or third best technique with a little difference.

Table 3 Results of AM, UO, OurMethod, optimal

\begin{tabular}{lllll}
\hline & AM & UO & OurMethod & Optimal \\
\hline Printtokens1 & 0.7680042 & 0.7481079 & 0.99733657 & 0.9996195 \\
Schedule1 & 0.5555975 & 0.5555975 & 0.9975262 & 0.9992872 \\
Tcas & 0.91280776 & 0.7558006 & 0.97557086 & 0.99732107 \\
Totinfo & 0.95960075 & 0.94910075 & 0.99412376 & 0.9983882 \\
Replace & 0.9149952 & 0.897003 & 0.94171196 & 0.9994296 \\
Printtokens2 & 0.58439314 & 0.58439314 & 0.999352 & 0.99973 \\
Schedule2 & 0.37809038 & 0.37809038 & 0.96434504 & 0.999098 \\
\hline
\end{tabular}

Table 2 Results of TS, AS, TB, $A B, T M$

\begin{tabular}{llllll}
\hline & TS & AS & TB & AB & TM \\
\hline Printtokens1 & 0.99802834 & 0.9163265 & 0.9975787 & 0.9161882 & 0.98367345 \\
Schedule1 & 0.7177778 & 0.6141719 & 0.73438156 & 0.7948008 & 0.6166457 \\
Tcas & 0.7682899 & 0.91280776 & 0.77508295 & 0.91280776 & 0.7720054 \\
Totinfo & 0.9693657 & 0.95960075 & 0.9783529 & 0.95960075 & 0.96949536 \\
Replace & 0.9093957 & 0.9149952 & 0.9148787 & 0.9149952 & 0.92015225 \\
Printtokens2 & 0.999514 & 0.8490077 & 0.9975787 & 0.9161882 & 0.98706627 \\
Schedule2 & 0.8682657 & 0.5071956 & 0.8934502 & 0.50654984 & 0.5740775 \\
\hline
\end{tabular}


Table 4 Ranking of all techniques based on their APFD results on subject programs

\begin{tabular}{lllllll}
\hline Schedule1 & Schedule2 & Printtokens1 & Printtokens2 & Tcas & Totinfo & Replace \\
\hline OurMethod & OurMethod & TS & TS & OurMethod & OurMethod & OurMethod \\
AB & TB & TB & OurMethod & AS & TB & TM \\
TB & TS & OurMethod & TB & AB & TM & AS \\
TS & TM & TM & TM & AM & TS & AB \\
TM & AS & AS & AB & TB & AS & AM \\
AS & AB & AB & AS & TM & AB & TB \\
AM & AM & AM & AM & TS & AM & TS \\
UO & UO & UO & UO & UO & UO & UO \\
\hline
\end{tabular}

\section{Conclusion}

In this paper, we described our approach which is actually a combination of three strategies: similarity-based, Total, and Additional. After that, we conducted an empirical study to evaluate our approach.To this end, we compared our technique with eight other techniques which six of them were based on Total and Additional strategies and all of them were run against Siemens benchmark programs.

As the analysis of the results demonstrates, it can be concluded that the proposed method is a technique that is more effective than the Additional and Total strategies, as well as more stable in terms of fault detection rate. As a future work, we plan to apply criteria other than Jaccard to measure the distance and similarity between test cases.

\section{Compliance with ethical standards}

Conflicts of interest The authors declare that they have no conflict of interest.

\section{References}

1. Yoo S, Harman M (2012) Regression testing minimization, selection and prioritization: a survey. Softw Test Verif Reliab 22(2):67-120

2. Rothermel G, Untch RH, Chu Ch, Harrold MJ (1999) Test case prioritization: an empirical study. In: Proceedings of IEEE international conference on software maintenance, 1999 (ICSM'99), pp 179-188
3. Zhang L, Hao D, Zhang L, Rothermel G, Mei H (2013) Bridging the gap between the total and additional test-case prioritization strategies. In: Proceedings of the 2013 international conference on software engineering. IEEE Press, pp 192-201

4. Yoo Sh, Harman M, Tonella P, Susi A (2009) Clustering test cases to achieve effective and scalable prioritisation incorporating expert knowledge. In: Proceedings of the eighteenth international symposium on software testing and analysis. ACM, pp 201-212

5. Jiang B, Zhang Zh, Chan WK, Tse TH (2009) Adaptive random test case prioritization. In: Proceedings of the 2009 IEEE/ACM international conference on automated software engineering. IEEE Computer Society, pp 233-244

6. Rothermel G, Untch RH, Chu Ch, Harrold MJ (2001) Prioritizing test cases for regression testing. IEEE Trans Softw Eng 27:929-948

7. Marré M, Bertolino A (2003) Using spanning sets for coverage testing. IEEE Trans Softw Eng 29(11):974-984

8. Xu R, Wunsch D (2005) Survey of clustering algorithms. IEEE Trans Neural Netw 16(3):645-678

9. Tan P et al (2007) Introduction to data mining. Pearson Education India, New Delhi

10. Do H, Elbaum S, Rothermel G (2005) Supporting controlled experimentation with testing techniques: an infrastructure and its potential impact. Empir Softw Eng 10(4):405-435

11. https://sir.csc.ncsu.edu/php/index.php. Last accessed 15 Oct 2018

12. Kim J, Porter A (2002) A history-based test prioritization technique for regression testing in resource constrained environments. In: Proceedings of the 24th international conference on software engineering, pp 119-129

13. Ledru Y, Petrenko A, Boroday S, Mandran N (2012) Prioritizing test cases with string distances. Autom Softw Eng 19(1):65-95

14. Li Z, Harman M, Hierons RM (2007) Search algorithms for regression test case prioritization. IEEE Trans Softw Eng 33(4):225-237

Publisher's Note Springer Nature remains neutral with regard to jurisdictional claims in published maps and institutional affiliations. 